\title{
Best-practice Indicators in Psoriatic Disease Care
}

\author{
Philip S. Helliwell, Guillaume Favier, Dafna D. Gladman, Enrique R. Soriano, \\ Bruce W. Kirkham, Laura C. Coates, Luis Puig, Wolf-Henning Boehncke, and Diamant Thaçi
}

ABSTRACT. Objective. In 2016, members of the Group for Research and Assessment of Psoriasis and Psoriatic Arthritis (GRAPPA), in collaboration with KPMG LLP (UK), conducted a study to measure care in psoriatic arthritis (PsA). A key finding was that centers do not usually have processes in place to measure the effect of improved quality of care. Our objectives were to identify and select best-practice indicators to enable PsA caregivers to assess and monitor the outcomes of specific initiatives aimed at improving care in 4 focus areas: (1) shortening time to diagnosis; (2) improving multidisciplinary collaboration; (3) optimizing disease management; and (4) improving disease monitoring.

Methods. (1) Structured review of scientific and grey literature to obtain evidence for a long list of 100 potential indicators across the 4 focus areas; (2) survey expert rheumatologists and dermatologists to review the long list and identify the most meaningful and feasible indicators for use in day-to-day practice; (3) consensus discussion to identify a shortlist of indicators based on predefined selection criteria; (4) electronic group discussion to refine definitions of shortlisted indicators and targets; and (5) review of the shortlisted indicators at the annual GRAPPA meeting in July 2018 to ensure the indicators meet the preliminary criteria.

Results. The expert group arrived at a consensus with a shortlist of 8 best-practice indicators across 4 key focus areas aligned with the patient pathway.

Conclusion. There were 8 evidence-based best-practice indicators and respective targets that were identified to enable the monitoring of quality of care and target improvements. (J Rheumatol Suppl. 2019 June;95:38-45; doi:10.3899/jrheum.190120)

Key Indexing Terms:

PSORIATIC ARTHRITIS GRAPPA

\section{PSORIASIS \\ QUALITY INDICATORS}

\section{QUALITY OF CARE DIAGNOSIS}

From the Leeds Institute of Rheumatic and Musculoskeletal Medicine, University of Leeds, Leeds; Bradford Hospitals National Health Service (NHS) Foundation Trust, Bradford; Global Strategy Group, Healthcare and Life Sciences, KPMG LLP, London; Guy's and St. Thomas' NHS Foundation Trust, Kings College London, London; University of Oxford, Oxford, UK; University of Toronto, Toronto, Ontario; Toronto Western Hospital, Toronto, Ontario, Canada; Rheumatology Unit, Internal Medicine Services, Hospital Italiano de Buenos Aires, Buenos Aires; University Institute Hospital Italiano de Buenos Aires, Buenos Aires; Argentina; Hospital de la Santa Creu I Sant Pau, Universitat Autònoma de Barcelona, Barcelona, Spain; Division of Dermatology and Venereology, Geneva University Hospitals, Geneva; Department of Pathology and Immunology, University of Geneva, Geneva, Switzerland; Research Institute and Comprehensive Center for Inflammation Medicine, University of Luebeck, Luebeck, Germany.

As part of the supplement series GRAPPA 2018, this report was reviewed internally and approved by the Guest Editors for integrity, accuracy, and consistency with scientific and ethical standards.

This project was funded by AbbVie.

P.S. Helliwell, DM, PhD, FRCP, Leeds Institute of Rheumatic and Musculoskeletal Medicine, University of Leeds, and Bradford Hospitals NHS Foundation Trust; G. Favier, PhD, Global Strategy Group, Healthcare and Life Sciences, KPMG LLP; D.D. Gladman, MD, FRCPC, Professor of Medicine, University of Toronto; and Senior Scientist, Krembil Research Institute, Toronto Western Hospital, and co-Chair, GRAPPA Publication Committee; E.R. Soriano, MD, MSc, Rheumatology Unit, Internal Medicine Services, Hospital Italiano de Buenos Aires, and University Institute Hospital Italiano de Buenos Aires; B.W. Kirkham, MD, FRCP, FRACP, Consultant Rheumatologist, Guy's and St. Thomas' NHS Foundation Trust, Professor of Translational Rheumatology, Kings College London; L.C. Coates, MB ChB, MRCP, PhD, University of Oxford; L. Puig, MD, PhD, Hospital de la Santa Creu I Sant Pau, Universitat Autònoma de Barcelona; W.H. Boehncke, MD, Professor,
Chair, Division of Dermatology and Venereology, Geneva University Hospitals, and Department of Pathology and Immunology, University of Geneva; D. Thaçi, MD, Research Institute and Comprehensive Center for Inflammation Medicine, University of Luebeck.

Address correspondence to P.S. Helliwell, Leeds Institute of Rheumatic and Musculoskeletal Medicine, University of Leeds, Second Floor, Chapel Allerton Hospital, Harehills Lane, Leeds LS7 4SA, UK. E-mail: p.helliwell@leeds.ac.uk

Psoriatic arthritis (PsA) is a chronic inflammatory musculoskeletal disease that affects peripheral joints, entheses, axial skeleton, skin, and nails. PsA is also associated with other comorbidities, with the effect of the disease extending beyond skin and joint symptoms. PsA impairs physical and mental function, decreases quality of life, results in reduced work productivity, and leads to high rates of healthcare use $^{1,2}$.

In 2016, members of the Group for Research and Assessment of Psoriasis and Psoriatic Arthritis (GRAPPA), in collaboration with KPMG LLP (UK), conducted a study to measure PsA care at treatment centers around the world ${ }^{3}$. The study identified $10 \mathrm{key}$ challenges in the care of patients with PsA as well as practices to improve these challenges. The top 4 challenges were late referral and diagnosis, limited awareness of PsA among non-rheumatologists, a disparate approach to care, and an inadequate management of comorbidities. 
A range of practices was identified to address these challenges in 4 corresponding focus areas:

1. Shorten time to diagnosis to ensure patients receive timely and appropriate screening.

2. Improve multidisciplinary collaboration to ensure PsA patients receive appropriate care.

3. Optimize disease management to ensure level of care is adjusted as required.

4. Improve disease monitoring to improve management of comorbidities.

Although the study observed a range of practices that could improve care for patients at treatment centers globally, it also suggested that PsA treatment centers do not usually have processes or metrics in place to measure quality of care ${ }^{3}$.

The objective of our present study was therefore to develop a set of indicators to enable PsA caregivers in the rheumatology and dermatology setting to objectively assess delivery of care while focusing on the 4 challenges and treatment practices discussed above. Such a set of indicators would be the first step in developing an objective measure of the effect of interventions aimed at improving care and to target future efforts ${ }^{4}$.

\section{MATERIALS AND METHODS}

Ethical approval for this study was not sought.

PsA expert panel composition. To ensure practical relevance in a range of PsA care settings, this study was supported by a panel of international experts consisting of 5 rheumatologists and 3 dermatologists across 3 geographies (Europe, North America, and South America). The panelists were all GRAPPA members and were recruited to reflect treatment centers with leading care practice and a range of care environments.

Based on panel input, a set of principles was defined to guide the identification and definition of a set of quality-of-care indicators. Namely, that each indicator should be (1) relevant to the key challenges in PsA care, (2) evidence-based, (3) feasible and measurable to be of practical use to PsA treatment centers around the world in both the rheumatology and dermatology care settings, and (4) formulated in a clear, concise, and sufficiently general way. The study comprised 4 phases to achieve these objectives, which are highlighted in Figure 1 and described in detail below.

Phase 1 consisted of an integrative literature review to identify a long list of 100 potential indicators. In Phase 2, through an online survey, the expert panel assessed the long list based on predefined feasibility criteria to identify a set of 77 indicators that would be practical to collect at the centers. In Phase 3, in a consensus discussion, the expert panel evaluated the resulting 77 indicators, focusing on relevance and reliability, to arrive at a shortlist of 11 indicators. Finally, in Phase 4, through electronic group discussion, a list of 8 core indicators was refined and attributed targets based on evidence from literature.

Phase 1: Integrative literature review. The study used an integrative literature review approach to identify relevant literature as an evidence base for best-practice indicators. A search in PubMed and online search engines was complemented with literature suggestions from the expert panel. The review covered both academic articles and grey literature, such as relevant guidelines and policy documents, that discussed PsA, additional arthritides, and other chronic diseases. This structured approach has been used in similar studies in arthritis and nursing, and allows rapid triage to arrive at the most relevant literature ${ }^{5,6}$. Figure 2 summarizes the literature review process.

Academic literature search. A database search for articles in PubMed related to best-practice indicators using a combination of prioritized search terms
(Table 1) was conducted to identify existing best-practice indicators. Additional articles were retrieved through the citation-tracking of original articles and were based on recommendations from the expert panel.

Grey literature search. The publicly available grey literature was examined by applying prioritized search terms in search engines (Table 1). Non-English-language references were excluded unless there was sufficient explanatory text in English.

The time allotted for the grey literature search was $1 \mathrm{~h}$ or until saturation was reached, whichever came first. Saturation was defined as not identifying new literature to include in analysis for $30 \mathrm{~min}$ or 5 consecutive search pages, whichever came first. The predefined time limit/saturation was set as a pragmatic limit while allowing a comprehensive search to be performed. After the academic and grey literature searches were completed, the literature list was supplemented through engagement with the expert panel and PsA care practitioners.

Overall, the searches of academic and grey literature returned about 3126 studies over the last 5 years, of which 288 were prioritized based on key search terms. Of the 288 studies, 183 were selected following a relevancy check by conducting a title review in which overlapping literature was eliminated. Subsequently, a review of the abstracts and additional findings from the expert panel and grey literature established a total of 143 relevant publications to be further evaluated. Finally, based on reviews of the full articles, 92 publications were considered relevant for identifying the indicators.

Identification of indicator long list. A review of the 92 selected academic and grey literature sources led to the identification of 100 potential indicators across the 4 focus areas. To support evaluation in subsequent phases of the study, an initial definition was constructed for each indicator regarding a measure with a unit, a target where available, and support by a rationale for measurement. To structure the analysis, the indicators were categorized into 3 groups: adoption of clinical practice, process measures (such as duration), and clinical and nonclinical outcomes. Figure 3 summarizes the distribution of indicators across these categories. For example, for "shorten time to diagnosis," a potential indicator in the category "process measures" was tentatively defined as "average duration from the onset of symptoms (such as joint pain, skin manifestations, etc.) to a diagnosis of PsA," measured in months, with a target of less than 5 years. In the following phases, the definition of selected indicators was refined iteratively based on the expert panel's review.

Phase 2: Expert panel survey to assess indicator feasibility. In Phase 2, the panel of 7 experts was asked to complete a survey to identify the most feasible indicators for use in day-to-day practice. For each of the long-listed 100 potential indicators, the panel assessed whether data required to measure the indicator would be available and whether the cost to retrieve these data was acceptable. The decision process to retain an indicator for detailed evaluation is outlined in Figure 4. Specifically, an indicator would be retained if: 1. The majority of experts (at least 4 out of 7) agreed that the indicator is currently captured or can easily be captured; or

2. Two or more experts agree that the indicator is currently captured, and 3 or more agree it could be easily captured in the future.

Of the 100 potential indicators from the initial long list, 77 were retained based on feasibility. Of the 23 indicators, 16 were discarded based on limited feasibility to collect the underlying data and were clinical and nonclinical outcomes. A primary concern regarding outcomes-related data was the ability to aggregate data either from patient reporting or health records in line with the effort reported in other studies required to collect patientreported effect of PsA on quality of life ${ }^{1}$.

Phase 3: Expert panel consensus discussion. In Phase 3, a group discussion involving the expert rheumatologists and dermatologists from the panel followed.

The panel assessed the 77 retained indicators in detail to identify a shortlist based on 2 criteria:

1. Relevance of the indicator for PsA best practice: Does measuring the indicator help evaluate delivery of care to identify potential for improvement?

Personal non-commercial use only. The Journal of Rheumatology Copyright $\subset$ 2019. All rights reserved. 


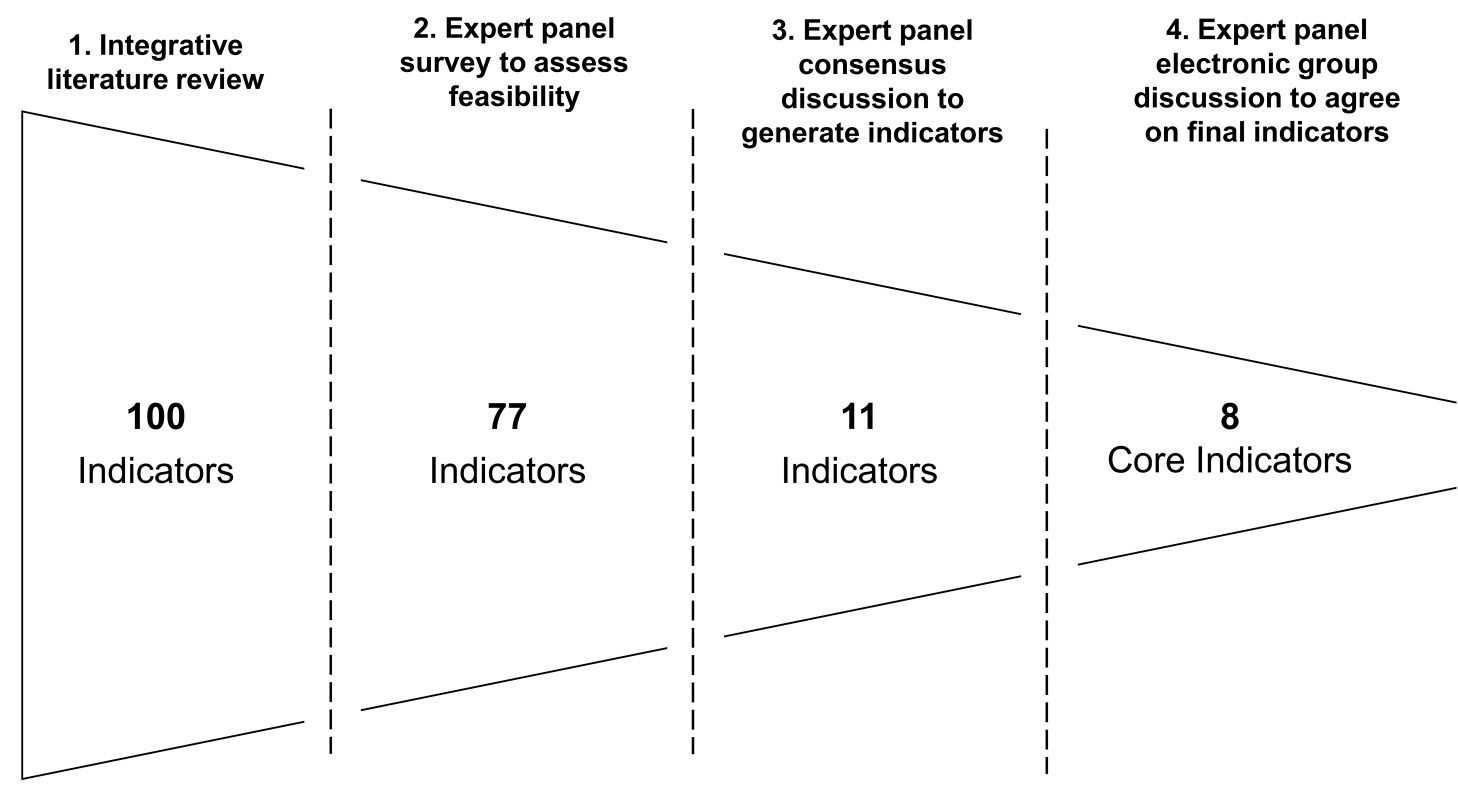

Figure 1. Study approach to identify PsA best-practice indicators that are evidence-based and of practical use to treatment centers.

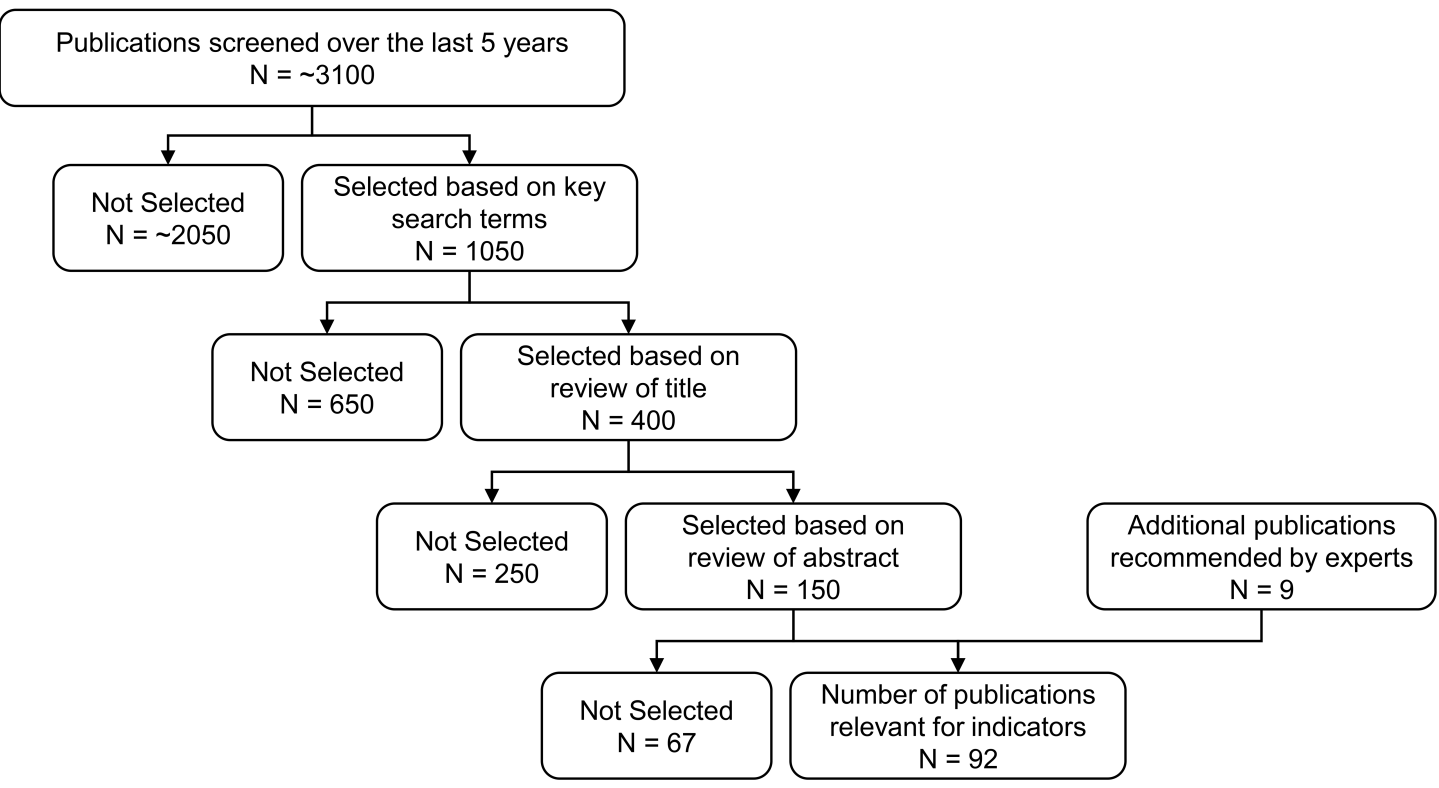

Figure 2. Studies identified in the integrative literature review.

Table 1. Prioritized search terms.

\begin{tabular}{lc}
\hline High-priority search term/group of search terms & Search Results (last 5 yrs) \\
\hline $\begin{array}{l}\text { Psoriatic arthritis / psoriatic arthritis management / management of psoriatic } \\
\text { arthritis / management of psoriasis }\end{array}$ & 3126 \\
Patient outcomes / patient-reported outcomes / patient outcome / patient-reported & 31 \\
outcome measures / patient satisfaction & 74 \\
Treatment / treatment challenges / treatment guidelines / guidelines / & 91 \\
treatment recommendations & 34 \\
Indicator / quality indicator / quality of care / quality of life / patient quality of life & 58 \\
Screening / diagnosis / delayed diagnosis / diagnostic delay / referrals / referral / symptoms & \\
Disease burden / multidisciplinary care / comorbidity & \\
\hline
\end{tabular}




\begin{tabular}{|c|c|c|c|c|c|}
\hline$\underbrace{\text { Focus }}_{\text {Sub-group }} \begin{array}{c}\text { area } \\
\end{array}$ & $\begin{array}{l}\text { Shorten } \\
\text { time to diagnosis }\end{array}$ & $\begin{array}{c}\text { Improve } \\
\text { multidisciplinary } \\
\text { collaboration }\end{array}$ & $\begin{array}{l}\text { Optimise disease } \\
\text { management }\end{array}$ & $\begin{array}{l}\text { Improve } \\
\text { disease } \\
\text { monitoring }\end{array}$ & Total \\
\hline $\begin{array}{l}\text { Adoption of } \\
\text { clinical practice }\end{array}$ & 5 & 6 & 11 & 7 & 29 \\
\hline Process & 18 & 3 & 9 & 2 & 32 \\
\hline Outcomes & 7 & 3 & 20 & 9 & 39 \\
\hline Total & 30 & 12 & 40 & 18 & 100 \\
\hline
\end{tabular}

Figure 3. Mapping of long list of potential quality indicators.

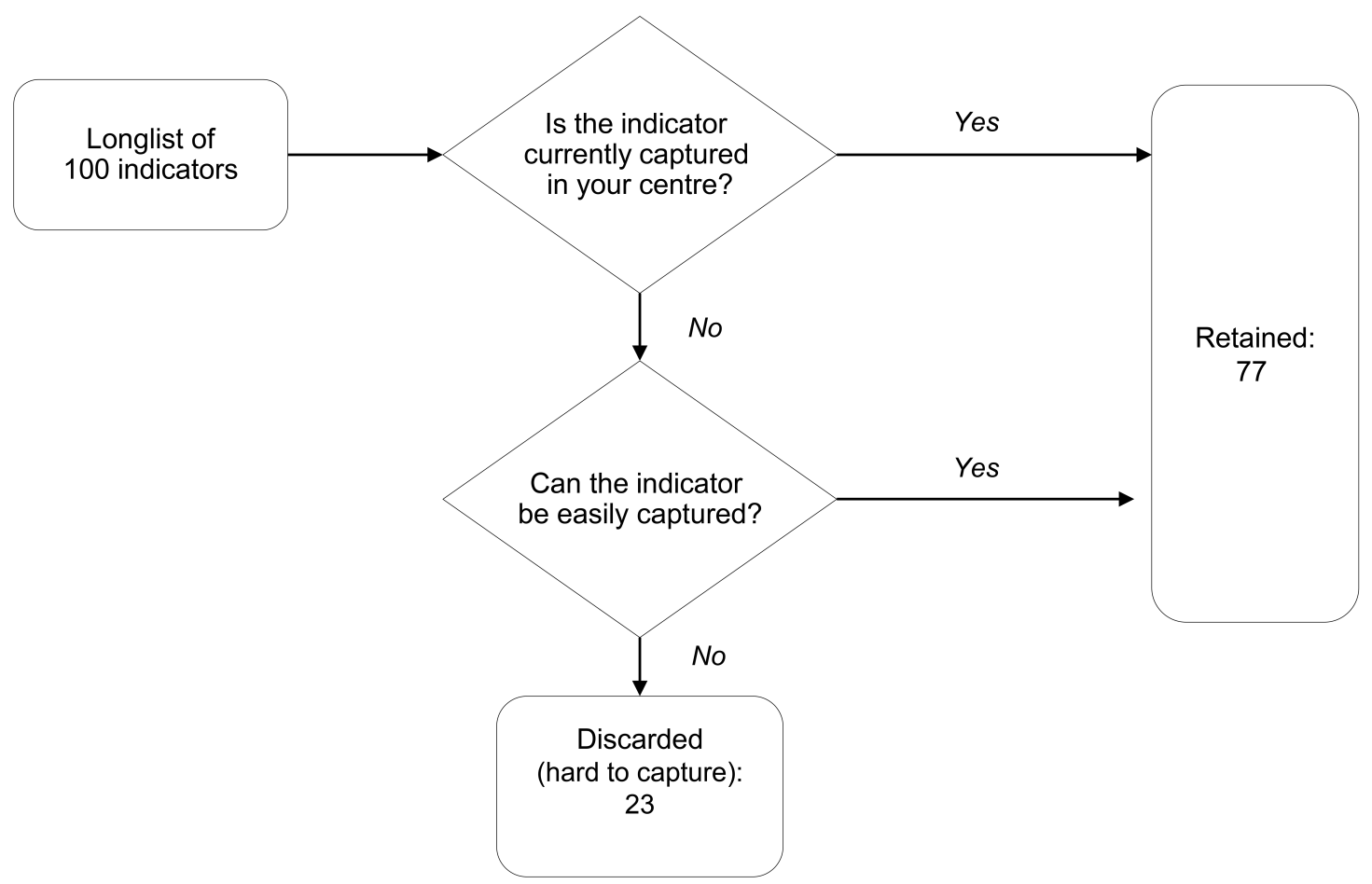

Figure 4. Feasibility expert panel survey.

2. Reliability of measurement: Does the indicator provide a consistent measure?

The panel also suggested the following key points to consider in the selection and definition of the indicators to ensure they would be of practical use in the PsA care setting:

- Retain as few indicators per focus area as possible (maximum of 4 per focus area).

- Ensure indicators are defined in a sufficiently general and simple way.

- Clearly communicate distinction between aggregate indicator (e.g., percent over all patients at treatment center) and the checklists (e.g., per individual patient) required to get individual data points.

Each of the 77 indicators was reviewed and comments were captured to refine the indicator definition and target, as well as to provide further evidence. The full list of 100 potential indicators was kept at hand to allow for reassessment of specific discarded indicators in case panel members deemed these sufficiently important. In particular, the indicator related to application of treat to target ${ }^{7}$, which had initially been discarded, was reincluded. Further, 2 new indicators that were not originally part of the top 100 were added based on panel suggestions.

The panel discussion led to a shortened list of 11 quality indicators for PsA care. The other 68 indicators were discarded because they were considered too detailed, could be combined into an overarching indicator (e.g., the duration of an intermediate step between presentation and diagnosis), or were not a direct or reliable measure of quality of PsA care.

Phase 4: Indicator generation. In Phase 4, a final electronic group discussion among the experts was conducted to refine the shortlist of 11 indicators, including their respective definitions and targets, and to provide supplementary evidence to support the rationale for inclusion in the final set. Specifically, the indicators were once more reviewed based on the guiding principles determined by the expert panel. Based on this review, 3 indicators were rejected because of limited feasibility or current lack of supporting evidence. For example, despite being a promising solution, a measure related to fast-tracking patients based on PsA risk score would first require a validated risk score to be established. 


\section{RESULTS}

The expert group arrived at a consensus with a shortlist of 8 indicators across each focus area, listed in Table 2.

Review at the annual GRAPPA meeting. In addition to the PsA expert panel, the final list of indicators was presented at the 2018 annual GRAPPA meeting, where about 150 participants, including expert rheumatologists, dermatologists, and patient research partners, evaluated the indicators and associated targets to assess their ability to improve PsA care. Figure 5 depicts the voting results from the workshop held at the meeting specific to the topic.

As part of the workshop, the 8 core indicators were reevaluated to seek patient perspective and input from a larger group of rheumatologists, dermatologists, and industry experts. Overall, the feedback was positive with a large majority of the participants agreeing with the relevance of presented indicators and respective targets to improve care for patients. The consensus view suggested the indicators would be useful measures for clinical practice, but that they should be repositioned from "quality indicators" to "best-practice indicators."

Detailed focused group discussions suggested that minor modifications to the existing indicators can provide greater clarity of measures. As a result, some of the best-practice indicators were further refined to enable ease of adoption in clinical practice, as shown in Table 3.

In addition to the refined list of core indicators included above, members at the GRAPPA workshop were also asked to evaluate the long list of 77 indicators that were originally reviewed by the expert panel. As a part of this exercise, members were asked to suggest additional potential indicators that may be valuable for PsA care. As a result, 7 additional indicators were put forward, as shown in Table 4.

\section{DISCUSSION}

We present here an initial set of concise indicators for best practice in PsA resulting from an integrative literature review, electronic survey, and consensus discussions among an international panel of dermatology and rheumatology experts, followed by a review at the annual GRAPPA meeting by a larger group of physicians and patients. These indicators are preliminary and can serve as benchmarks for delivering quality care in the clinical setting and may enhance the care of patients with psoriatic disease worldwide. It is clear that the indicators relate more to PsA than psoriasis, although it could be argued that they cut across these 2 specialties and may serve to monitor and enhance care for patients with both conditions.

We acknowledge a number of limitations to this study and highlight a number of potential next steps. First, patient involvement in the project occurred late. At the 2018 annual GRAPPA meeting, a patient voice at the outset may have changed the prioritizing of the indicators. Second, to ensure that the PsA best-practice indicators would be of practical use to healthcare professionals, in Phase 2 of this study only potential indicators that the panel deemed feasible to collect were retained. As a result, 23 indicators were discarded in Phase 2 because the panel presumed limited availability of data for these and/or effort required to collect these prohibitive. Because of this, potentially relevant indicators may have been discarded based on a subjective evaluation of data availability. A next step would be to review the discarded

Table 2. Best practice indicators for PsA.

1. Shorten time to diagnosis

Average duration from presentation to HCP to confirmed PsA diagnosis ${ }^{8}$

Percent of patients with psoriasis in a year who receive a PsA screening test (a suitable

validated tool such as PEST, CONTEST, or other questionnaires $)^{9}$

2. Improve multidisciplinary collaboration

Multidisciplinary PsA assessment is available $(\mathrm{Y} / \mathrm{N})^{10}$

Does the center provide suitable training for $\mathrm{HCP}$, nurses, etc., to increase awareness of PsA disease symptoms $(\mathrm{Y} / \mathrm{N})^{11}$

3. Optimize disease management

Average number of PsA evaluations done by HCP per patient in a year (depending on the specialty), assessing 6 core domains of PsA: musculoskeletal, skin, function, pain, patient's global assessment, and quality of life ${ }^{12}$

Percent of PsA patients on whom T2T strategy is applied ${ }^{7}$

4. Improve disease monitoring

Percent of PsA patients who received full disease assessment for comorbidities

(e.g., comorbidity index) at least once every year ${ }^{13}$

Availability of short-term, unscheduled appointments $(\mathrm{Y} / \mathrm{N})^{14}$

$$
<6 \operatorname{mos}
$$

PsA screening test to be conducted at least once a year

Multidisciplinary collaboration should be available in centers $100 \%$ of staff should have followed suitable training on PsA each year

1-2 evaluations per year to monitor disease activity

All patients with new-onset disease should be offered a T2T strategy

All patients should have at least an annual assessment for comorbidities

Maximum wait time for unscheduled appointment should be 2 weeks

HCP: healthcare providers; PsA: psoriatic arthritis; PEST: Psoriasis Epidemiology Screening Tool; CONTEST: CONTEST [study] screening tool; T2T: treat to target.

Personal non-commercial use only. The Journal of Rheumatology Copyright $\odot$ 2019. All rights reserved. 


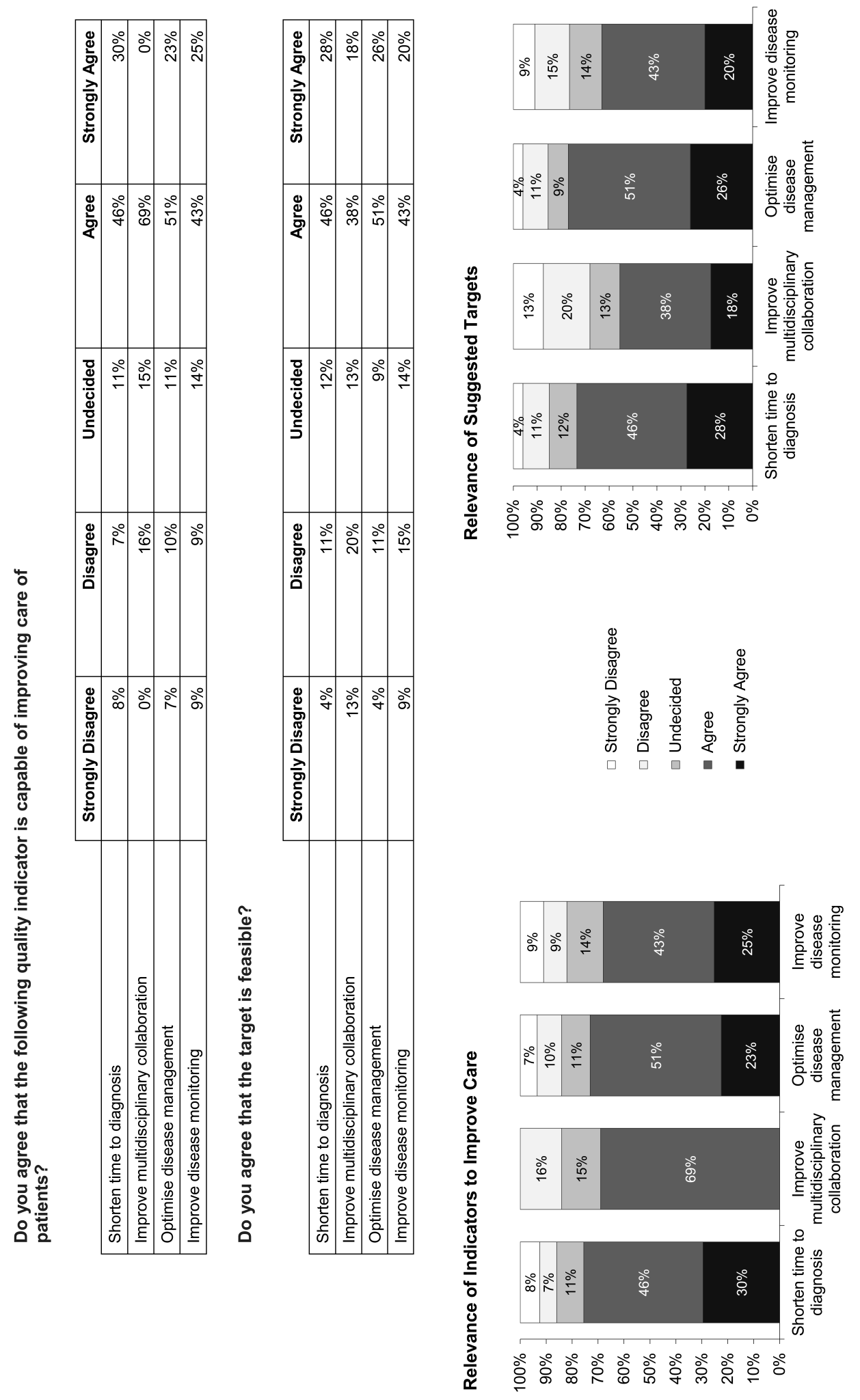

Figure 5. Voting results, annual GRAPPA meeting. GRAPPA: Group for Research and Assessment of Psoriasis and Psoriatic Arthritis. 
Table 3. Refined list of best practice indicators.

1. Shorten time to diagnosis

Average duration from presentation to HCP to confirmed PsA diagnosis ${ }^{8}$

Percent of patients with psoriasis in a year who receive PsA screening tests (clinical or a suitable validated tool such as PEST, CONTEST, or other questionnaire) ${ }^{9}$

2. Improve multidisciplinary collaboration

Multidisciplinary PsA assessment is available $(\mathrm{Y} / \mathrm{N})^{10}$

Does the center provide training to increase awareness of symptoms associated

with psoriatic disease and train $\mathrm{HCP}$, nurses, etc., to conduct disease assessment? ${ }^{11}$

3. Optimize disease management

Average number of evaluations conducted in a year for psoriatic patients who have visited HCP more than once, assessing 6 core domains of PsA: musculoskeletal, skin, function, pain, patient's global assessment, and quality of life $\mathrm{e}^{12}$

Percent of new PsA patients who are treated using the T2T strategy or with an agreed treatment goal ${ }^{12}$

4. Improve disease monitoring

Percent of psoriatic disease patients who received full disease assessment

at least once every year (e.g., comorbidity index can be leveraged

to conduct disease assessment $)^{13}$

Availability of short-term unscheduled appointments for new patients $(\mathrm{Y} / \mathrm{N})^{14}$
$<6 \operatorname{mos}$

PsA screening test to be conducted at least once a year

Multidisciplinary collaboration should be available in centers $100 \%$ of staff should have followed suitable training on PsA each year

1-2 evaluations per year to monitor disease activity

All patients with new-onset disease should be offered a T2T strategy

All patients should have at least an annual assessment for comorbidities

Maximum wait time for unscheduled appointment should be 2 weeks

HCP: healthcare providers; PsA: psoriatic arthritis; PEST: Psoriasis Epidemiology Screening Tool; CONTEST: CONTEST [study] screening tool; T2T: treat to target.

Table 4. Additional potential indicators that may be relevant to measure.

Additional Indicators Suggested at GRAPPA

1. Shorten time to diagnosis

Average time from the first contact with HCP to a rheumatology or dermatology referral

Average time from the HCP referral to a dermatology appointment

Average time from the HCP referral to a rheumatology appointment

2. Improve multidisciplinary collaboration

Percent of PsA staff at the center who participated in at least 1 PsA-related training session in last year

Number of educational and collaborative meetings organized between dermatologist, rheumatologist, and other specialists (such as cardiologist,

ophthalmologist, etc.) in each quarter

Percent attendance at the multidisciplinary networking and community-based meetings every quarter

4. Improve disease monitoring

Percent of PsA patients on whom a comprehensive risk/benefit and longterm side effects assessment is conducted in a year (in followup period)

GRAPPA: Group for Research and Assessment of Psoriasis and Psoriatic Arthritis; HCP: healthcare providers; PsA: psoriatic arthritis.

indicators for relevance, followed by a pilot study to assess data availability. Additionally, for best-practice indicators to be useful and meaningful, they must also depict an array of attributes: feasibility, acceptability, reliability, and validity. In this study we have tested indicators based only on feasibility to capture data and availability of target evidence.

The current definition of targets for some of the 8 indicators could benefit from tailoring to current practice. For example, it may not be feasible to achieve annual PsA screening for $100 \%$ of patients with psoriasis, and a different target may be more appropriate, although in general, patient research partners were reluctant to reduce the targets, to force the maintenance of higher standards of quality care.

In some cases, indicators were not used because they were not yet evaluated in the literature or because there is no consensus on desired clinical practice. As PsA care is further standardized and new information from research is available, it may be worth revisiting and expanding the shortlist of indicators.

The indicators are currently aimed at supporting best- practice care for patients with PsA, to aid healthcare professionals by setting up a measuring framework and to give them the opportunity to identify gaps in the quality of their care that otherwise might remain undetected. The indicators may also benefit from additional, more targeted measures of care. For example, regarding delays in time to diagnosis, the indicator currently measures the overall time to diagnosis from first presentation to healthcare providers. Modification of the indicator may unravel delay along specific steps of the patient pathway, which may also be 
useful to record to highlight further opportunities for improvement.

This project has identified 8 best-practice indicators for PsA care across 4 practice areas. The respective targets are evidence-based, feasible, measurable, and meaningful for PsA care providers. These indicators may be used in practice to further assess the delivery of care and to allow for the identification of areas for improvement.

\section{ACKNOWLEDGMENT}

The authors thank GRAPPA's President, Dr. Kristina Callis Duffin, and fellow members Drs. April Armstrong, Alberto Cauli, Lihi Eder, Oliver FitzGerald, Niti Goel, Joseph Merola, Cheryl Rosen, Ruben Querio Silva, Carmel Stober, and William Tillett for facilitating the workshop at GRAPPA's 2018 annual meeting. The authors are also grateful to GRAPPA Patient Research Partners Rodrigo Firmino, Suzanne Grieb, and Chris Lindsay (Patient Research Partner employed by Amgen Inc., Thousand Oaks, California, USA) for their input and contribution to the work.

\section{REFERENCES}

1. Gladman DD, Antoni C, Mease P, Clegg DO, Nash P. Psoriatic arthritis: epidemiology, clinical features, course, and outcome. Ann Rheum Dis 2005;64 Suppl 2:ii14-7.

2. Kavanaugh A, Helliwell P, Ritchlin CT. Psoriatic arthritis and burden of disease: patient perspectives from the population-based Multinational Assessment of Psoriasis and Psoriatic Arthritis (MAPP) survey. Rheumatol Ther 2016;3:91-102.

3. Favier G, Gladman DD, Merola JF, Armstrong AW, Boehncke WH, Helliwell PS. Benchmarking care in psoriatic arthritis - The QUANTUM Report: a report from the GRAPPA 2016 annual meeting. J Rheumatol 2017;44:674-8.

4. KPMG. Improvement in the management of psoriatic arthritis. [Internet. Accessed February 21, 2019.] Available from: www.grappanetwork.org/assets/PDFs/psa\%20care\%20report\% 20-\%20final.pdf
5. Whittemore R, Knafl K. The integrative review: updated methodology. J Adv Nurs 2005;52:546-53.

6. Barber CE, Patel JN, Woodhouse L, Smith C, Weiss S, Homik J, et al. Development of key performance indicators to evaluate centralized intake for patients with osteoarthritis and rheumatoid arthritis. Arthritis Res Ther 2015;17:322.

7. Smolen JS, Braun J, Dougados M, Emery P, Fitzgerald O, Helliwell $\mathrm{P}$, et al. Treating spondyloarthritis, including ankylosing spondylitis and psoriatic arthritis, to target: recommendations of an international task force. Ann Rheum Dis 2014;73:6-16.

8. Haroon M, Gallagher P, FitzGerald O. Diagnostic delay of more than 6 months contributes to poor radiographic and functional outcome in psoriatic arthritis. Ann Rheum Dis 2015;74:1045-50.

9. NICE. National Institute for Health and Care Excellence. Psoriasis: assessment and management. CG153. [Internet. Accessed February 21, 2019.] Available from: www.nice.org.uk/Guidance/CG153

10. Urruticoechea-Arana A, Serra Torres M, Hergueta Diaz M, Gonzalez Guerrero ME, Farinas Padron L, Navarro Martin S, et al. Experience and satisfaction with a multidisciplinary care unit for patients with psoriasis and psoriatic arthritis. Reumatol Clin 2017; S1699-258X:30183-3.

11. Betteridge N, Boehncke WH, Bundy C, Gossec L, Gratacos J, Augustin M. Promoting patient-centred care in psoriatic arthritis: a multidisciplinary European perspective on improving the patient experience. J Eur Acad Dermatol Venereol 2016;30:576-85.

12. Combe B, Landewe R, Daien CI, Hua C, Aletaha D, Alvaro-Gracia JM, et al. 2016 update of the EULAR recommendations for the management of early arthritis. Ann Rheum Dis 2017;76:948-59.

13. El Miedany Y, El Gaafary M, Youssef S, Bahlas S, Hegazi M. Psoriatic arthritis comorbidity index: development and validation of a new specific tool for classifying prognostic comorbidity in psoriasis and psoriatic arthritis patients. Rheumatol Orthop Med 2017;2:1-7.

14. Lebwohl MG, Kavanaugh A, Armstrong AW, Van Voorhees AS. US perspectives in the management of psoriasis and psoriatic arthritis: patient and physician results from the population-based Multinational Assessment of Psoriasis and Psoriatic Arthritis (MAPP) survey. Am J Clin Dermatol 2016;17:87-97. 